\section{Kinetochores distinguish GTP from GDP forms of the microtubule lattice}

\author{
Fedor F. Severin ${ }^{\star}$, Peter K. Sorger $\dagger$ \& Anthony A. Hyman* \\ ${ }^{\star}$ European Molecular Biology Laboratory, Postfach 10.2209, D-69012 Heidelberg, \\ Germany \\ $\dagger$ Department of Biology, Massachusetts Institute of Technology, Cambridge, \\ Massachusetts 02139, USA
}

During prometaphase in mitotic cell division, chromosomes attach to the walls of microtubules and subsequently move to microtubule ends, where they stay throughout mitosis ${ }^{1,2}$. This end-attachment seems to be essential for correct chromosome segregating. However, the mechanism by which kinetochores, the multiprotein complexes that link chromosomes to the microtubules of the mitotic spindle ${ }^{3,4}$, recognize and stay attached to microtubule ends is not understood. One clue comes from the hydrolysis of GTP that occurs during microtubule polymerization. Although tubulin dimers must contain GTP to polymerize, this GTP is rapidly hydrolysed following the addition of dimers to a growing polymer. This creates a microtubule consisting largely of GDP-tubulin, with a small cap of GTP-tubulin at the end $d^{5}$. It is possible that kinetochores distinguish the different structural states of a GTP- versus a GDP-microtubule lattice. We have examined this question in vitro using reconstituted kinetochores from the yeast Saccharomyces cerevisiae. We found that kinetochores in vitro bind preferentially to GTP- rather than GDPmicrotubules, and to the plus-end preferentially over the lattice. Our results could explain how kinetochores stay at microtubule ends and thus segregate chromosomes correctly during mitosis in vivo. This result demonstrates that proteins exist that can distinguish the GTP conformation of the microtubule lattice.

Chromosome segregation in mitosis is mediated by kinetochores. To monitor the relative attachment of kinetochores to the GDP versus the GTP parts of a microtubule lattice, we constructed segmented microtubules containing regions of both forms of tubulin. As GTP hydrolysis occurs within seconds of microtubule polymerization (the rate of hydrolysis exceeds $4 \times 10^{-2} \mathrm{~s}^{-1}$ (refs $6-8$ ), we made the GTP-microtubule parts using a slowly hydrolysable analogue, GMPCPP. In previous experiments, the hydrolysis rate of GMPCPP has been measured as $4 \times 10^{-7} \mathrm{~s}^{-1}$ (ref. 9). Microtubules were nucleated from Oregon-green fluorescent GMPCPP tubulin (Fig. 1a, green circles). We measured the rate of hydrolysis under these conditions, confirming that less than $4 \%$ of GMPCPP was hydrolysed over $4 \mathrm{~h}$ of polymerization (data not shown). GMPCPP microtubules were further elongated by addition of rhodamine GTP tubulin (red circles) which, hydrolysing to GDP after polymerization, creates a GDP segment. To stabilize the GDP regions, microtubules were capped by further addition of GMPCPP tubulin.

To test whether kinetochores can distinguish between GTP- and GDP-tubulin parts of microtubules, we reconstituted kinetochores from $S$. cerevisiae in vitro ${ }^{10}$. We began by attaching $S$. cerevisiae centromere DNA to rhodamine-labelled latex beads (Fig. 1a). The beads were incubated in yeast extract, and the bead-bound kinetochore complexes were then mixed with microtubules. Bead attachment to microtubules was monitored by fluorescence microscopy. As previously shown ${ }^{10}$, kinetochore complexes assemble on the beads and attach to microtubules.

We monitored the interaction of the reconstituted kinetochores with GMPCPP and GDP parts of microtubule lattice, ignoring any attachment to microtubule ends (see below). A microtubule with a kinetochore bead bound to a GMPCPP (green) region of the microtubule is shown in Fig. 2a; the GDP-tubulin region and the bead itself are red. After counting the beads bound to GMPCPP or GDP-tubulin and normalizing by the lengths of the microtubules, we found that $92 \pm 4 \%$ of the kinetochores bound to the GMPCPP regions of the microtubules (Fig. 2b). Thus an in vitro reconstituted kinetochore can distinguish the GTP state from the GDP state of a microtubule lattice $\left(P<0.0001, t\right.$-test $\left.{ }^{11}\right)$.

We have shown previously that GMPCPP microtubules have a different structure from GDP microtubules ${ }^{12}$. We wondered whether kinetochores were recognizing a unique feature of GMPCPP tubulin or rather a structural motif of the microtubule lattice. Electron microscopy data show that the structure of GDP and GMPCPP microtubules are different ${ }^{12}$. Addition of taxol changes GDP microtubules so that their lattice structure resembles that of GMPCPP microtubules ${ }^{13}$. We therefore tested whether kinetochores can bind to a GDP microtubule stabilized with taxol. We made capped microtubules (Fig. 1b) and split them into two portions. We added taxol to one portion and then mixed both portions with kinetochore beads. The percentage of the beads bound to GMPCPP versus GDP tubulin are shown in Fig. 1, and demonstrate that, after taxol was added, kinetochores bind equally to GDP and GMPCPP sections of microtubules $(P>0.2$, chisquared test $\left.{ }^{11}\right)$. Thus it seems that kinetochores recognize a structural motif of microtubules that correlates with microtubule stabilization.

In these experiments we noticed that several beads were bound to the ends of microtubules, but ignored them for the lattice-binding experiments. However, kinetochore microtubules of mitotic spindles have their minus ends at the spindle poles, and their plus ends bound to kinetochores ${ }^{14}$. Therefore, a kinetochore might be expected to recognize the plus-ends of microtubules. To quantify the degree to which kinetochores bound to microtubule plus-ends, we polymerized polarity-marked microtubules consisting only of
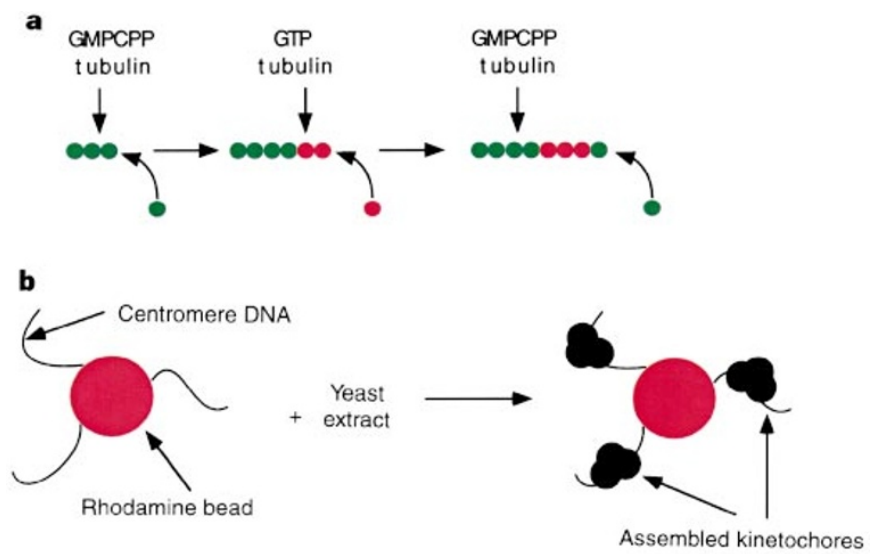

Figure 1 a, Preparation of segmented microtubules. Tubulin labelled with Oregon green was mixed with GMPCPP (green circles). The mixture was incubated at $37^{\circ} \mathrm{C}$ to allow tubulin to polymerize. The GMPCPP microtubules thus formed were diluted into rhodamine-labelled tubulin (red circles) in the presence of GTP. GTPtubulin polymerized at the plus-ends of the GMPCPP fragments. GTP-tubulin in polymerized state rapidly hydrolyses the associated GTP molecule into GDP. The GMPCPP-GDP microtubules were diluted into Oregon green-labelled tubulin in the presence of GMPCPP. After polymerization of GMPCPP-tubulin, the microtubules consisted of two GMPCPP fragments at the ends with GDP-tubulin fragments between them. b, In vitro reconstitution of $S$. cerevisiae kinetochore. The centromere DNA is attached to rhodamine-labelled latex beads by a biotinstreptavidin bond. The beads are incubated in the yeast extract and the kinetochores assemble on the DNA 
GMPCPP tubulin, with a short red minus-end and a long red plusend, separated by a green segment (Fig. 3a). We mixed kinetochore beads with polarity-marked microtubules and plotted the position of beads along microtubules. The distribution (Fig. 3b) shows that kinetochore beads preferentially bind the microtubule plus-end compared to the rest of the microtubule $\left(P<0.01, t\right.$-test $\left.{ }^{11}\right)$.

Our results show that in vitro reconstituted kinetochores from $S$. cerevisiae bind preferentially to GMPCPP microtubules rather than to GDP microtubules, and have a high affinity for microtubule plusends. The binding properties of these reconstituted kinetochores are similar to those of isolated mini-chromosomes from yeast ${ }^{15}$, and have many of the properties expected of a kinetochore in vivo ${ }^{10}$. In a different system, a difference in stability of mammalian chromosome-attached microtubule plus-ends compared to minus-ends has been demonstrated ${ }^{31}$, suggesting a difference in binding affinity of kinetochores to microtubule plus-ends, although the issue of whether kinetochores distinguish GTP from GDP lattices was not addressed. Our experiments were performed in vitro as such a detailed level of analysis is not possible in vivo. In vivo, a simple attachment of the microtubule wall matures to a complex end-on attachment in kinetochores of complex eukaryotes, although this yeast has not been seen in vivo. Future investigation will determine which aspects of the in vivo state have been reconstituted in vitro.

The mechanisms by which kinetochores stay at microtubule ends in vivo has long been a mystery, but our results suggest that kinetochores could use the structural features of the microtubule lattice to recognize and stay at microtubule ends. This is surprising because most data suggest that there is little GTP at microtubule ends $s^{6,16}$. We can imagine at least two possibilities. First, most data on rates of GTP hydrolysis in microtubules are from in vitro studies. It is quite possible that the rate of GTP hydrolysis in microtubules in vivo is different or variable, creating a longer GTP cap. The second possibility is that kinetochores bound to microtubule ends could stabilize the GTP state, inhibiting hydrolysis.

a

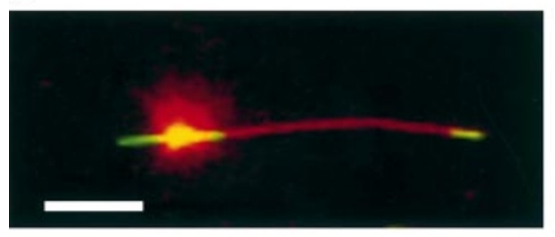

b
GMPCPP

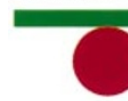

$92 \pm 4 \%$

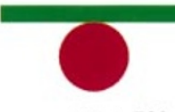

$57 \pm 5 \%$
GDP

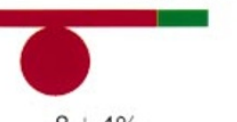

$8 \pm 4 \%$
With taxol

No taxol

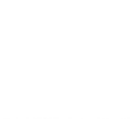

With taxol
Figure 2 a, Photograph of the capped microtubule with the bead bound to the GMPCPP tubulin region. GMPCPP-tubulin is labelled green, GDP-tubulin and the kinetochore bead are labelled red. Scale bar, $5 \mu \mathrm{m}$. b. The distribution of kinetochore beads between the regions in the presence and in the absence of taxol. In total we counted 75 beads bound to microtubules, and averages over three experiments were as follows: beads bound to GMPCPP region, $92 \pm 4 \%$; beads bound to GDP region, $8 \pm 4 \%$. Two more experiments have been done with kinetochore beads binding to capped microtubules in the presence of taxol. In total we counted 64 beads bound, and averages of three experiments were: beads bound to GMPCPP region, $57 \pm 5 \%$; beads bound to GDP region, $63 \pm 5 \%$.
What are the kinetochore proteins that interact with microtubules? It is known that a complex of four proteins binds to centromere DNA in vitro and is required for kinetochore function in $v_{i v o^{17-20}}$. It has been shown that the complex is necessary but not sufficient for kinetochores to bind to microtubules in vitro ${ }^{10,21}$, suggesting that other, as-yet unidentified, factors recognize and stabilize the GTP form of microtubules. It is possible that these proteins have homology to the tip attachment complexes in Tetrahymena flagella ${ }^{22,23}$, and one of the Tetrahymena proteins also seems to associate with mammalian kinetochores ${ }^{22,23}$.

In vivo, when kinetochores move away from poles, microtubules polymerize $^{24}$. We propose that proteins that recognize the GTP form of microtubules provide a powerful mechanism to couple movement and polymerization. If we imagine a kinetochore bound to a microtubule end using such a protein, the kinetochore would be tightly bound (Fig. 4a). Further polymerization would trigger GTP hydrolysis, weakening the interaction (Fig. 4b), allowing the kinetochore to move again until it re-establishes contact with the GTP end (Fig. 4c). Such movement could either be by random diffusion along the microtubule or by microtubule-based motors. We have no evidence yet for plus-end motors on yeast kinetochores, but plus-end motors have been localized to kinetochores in mammalian cells ${ }^{25}$. Such interactions between motors and proteins that recognize structural features of microtubules could act as a general mechanism by which organelles can be positioned on microtubules.

Our results also have implications for the control of microtubule behaviour. Microtubules turn over by switching constantly between growing and shrinking states ${ }^{26,27}$, and the rate at which microtubules interconvert between growing and shrinking is known to be regulated, but the mechanisms are obscure ${ }^{28,29}$. If other microtubule-associated proteins, like the putative kinetochore protein, bind preferentially to GTP tubulin, these proteins could bind to microtubule ends and therefore affect the microtubule

a
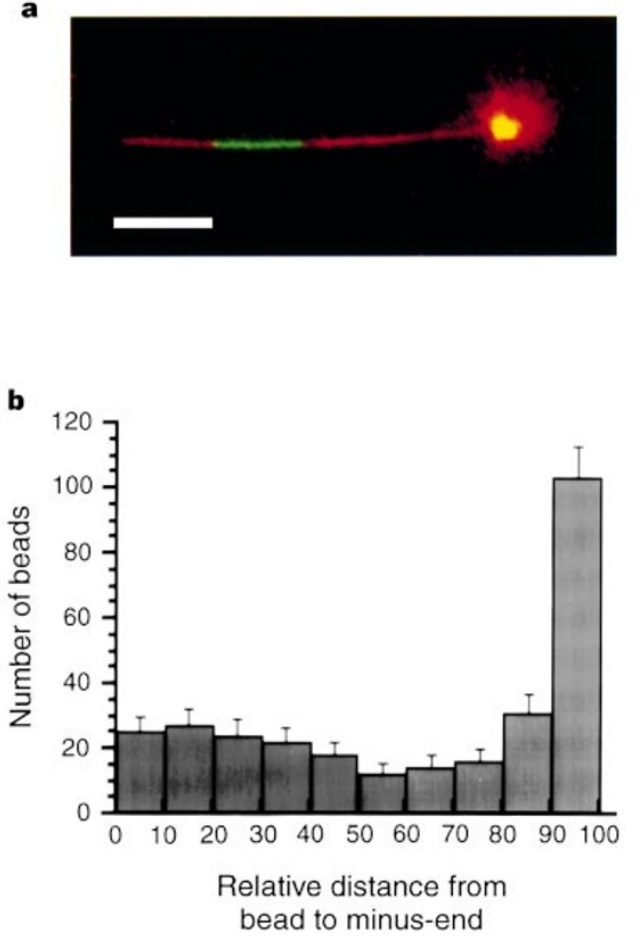

Figure $\mathbf{3} \mathbf{a}$, The bead bound to the plus-end of the GMPCPP microtubule. Scale bar, $5 \mu \mathrm{m}$. b. The distribution of the kinetochore beads bound to GMPCPP microtubules. Three independent experiments have been done, and 292 beads counted. The results of the experiments were similar and were added together. Error bars are square roots of the number of beads in each column. 


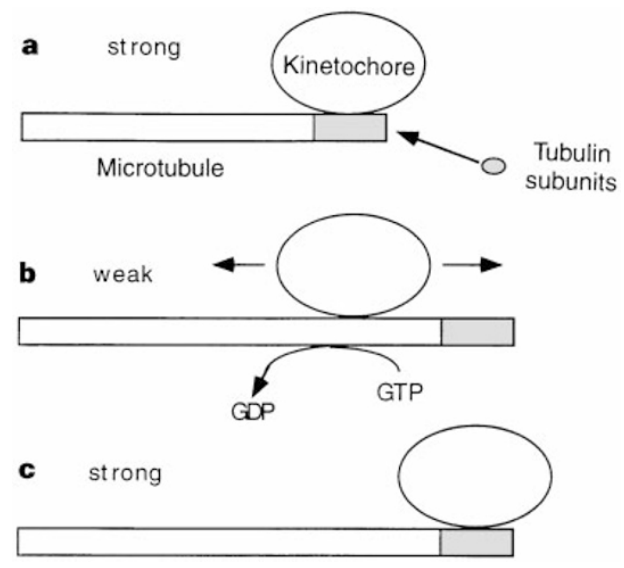

Figure 4 Model for kinetochore behaviour on mitotic-spindle microtubule. a, The kinetochore binds to the GTP part of the microtubule. $\mathbf{b}$, The microtubule polymerizes by end-on addition of GTP-tubulin subunits and GTP hydrolyses, weakening the attachment of the kinetochore to the microtubule. c. The kinetochore moves to the end of the microtubule, where it reattaches to GTPtubulin.

dynamics at substoichiometric amounts. Although most microtubule-associated proteins so far isolated bind along microtubule lattices, it is important to remember that all such preparations have been made using taxol-stabilized microtubules. Because we have shown that taxol mimics the GTP state of microtubules, it remains possible that known microtubule-associated proteins can distinguish between GTP and GDP states of microtubules.

\section{Methods}

In vitro reconstitution of $\boldsymbol{S}$. cerevisiae kinetochores. Yeast extracts were made as described ${ }^{10}$ except that yeast cells were broken in a buffer containing (in $\mathrm{mM}) 50$ bis-Tris-propane, pH 7.0, $100 \beta$-glycerophosphate, 2 EDTA, 2 EGTA, $200 \mathrm{KCl} 10 \%$ glycerol. CEN3 DNA was attached to rhodamine latex beads as described $^{10}$. To assemble the kinetochore complexes on the beads, $200 \mu \mathrm{g}$ of extract was added to $10^{7}$ beads and $2 \mu \mathrm{g}$ of sonicated salmon sperm DNA in kinetochore buffer (10 mM HEPES, pH 8.0, $6 \mathrm{mM} \mathrm{MgCl}_{2}, 10 \%$ glycerol) and incubated for $30 \mathrm{~min}$ at room temperature.

Preparation of capped microtubules. Tubulin was prepared and labelled with rhodamine or Oregon green (Molecular Probes, Eugene) as described ${ }^{30}$. $1 \mu \mathrm{M}$ Oregon green tubulin was added to $0.5 \mathrm{mM} \mathrm{GMPCPP}$ in BRB80 $(80 \mathrm{mM}$ potassium PIPES, pH $6.8,1 \mathrm{mM} \mathrm{MgCl} 2,1 \mathrm{mM} \mathrm{EGTA)} \mathrm{at} 37^{\circ} \mathrm{C} .1 \mu \mathrm{M}$ Oregon green tubulin was added to the mixture every $30 \mathrm{~min}$, for $2-3 \mathrm{~h}$, creating GMPCPP microtubules with an average length of $6.5 \mu \mathrm{m}$. Then $2.0 \mu \mathrm{l}$ of $0.1 \mathrm{mM}$ GTP, $10 \mu \mathrm{M}$ tubulin, $5 \mu \mathrm{M}$ rhodamine tubulin in BRB80 were preincubated for $10 \mathrm{~min}$ on ice and pre-warmed for $30 \mathrm{~s}$ at $37^{\circ} \mathrm{C} .2 \mu \mathrm{l}$ Oregon green microtubules was added to the mixture and GTP tubulin was allowed to polymerize for $20 \mathrm{~min}$. Under these conditions, $94 \%(n=69)$ of the GMPCPP microtubules nucleated GTP microtubules. $40 \mu \mathrm{l}$ of $0.5 \mu \mathrm{M}$ Oregon green tubulin, $0.5 \mathrm{mM}$ GMPCPP in BRB80 were preincubated on ice for $10 \mathrm{~min}$, pre-warmed at $37^{\circ} \mathrm{C}$ for $1 \mathrm{~min}$ and mixed with $0.5 \mu \mathrm{l}$ of the GMPCPP-GTP microtubules, giving a final GTP concentration of $1 \mu \mathrm{M}$ and a GMPCPP concentration of $500 \mu \mathrm{M}$. The mixture was replenished with $0.5 \mu \mathrm{M}$ Oregon green tubulin after $1 \mathrm{~h}$ of incubation at $37^{\circ} \mathrm{C}$ and left at $37^{\circ} \mathrm{C}$ for $30 \mathrm{~min}$. For the taxol experiment, $20 \mu \mathrm{l}$ of microtubules was mixed with either $20 \mu \mathrm{l}$ of BRB80 or $20 \mu \mathrm{l}$ of $50 \mu \mathrm{M}$ taxol in BRB80. Taxol microtubules were centrifuged for $5 \mathrm{~min}$ at 50,000 r.p.m. in a TLA 100.1 rotor and resuspended in $15 \mu \mathrm{l}$ of BRB80 with or without $10 \mu \mathrm{M}$ taxol.

Preparation of GMPCPP microtubules. $1 \mu \mathrm{M}$ Oregon green tubulin was added to $0.5 \mathrm{mM}$ GMPCPP in BRB80 (80 mM potassium PIPES, $\mathrm{pH} 6.8,1 \mathrm{mM}$ $\mathrm{MgCl}_{2}, 1 \mathrm{mM}$ EGTA) at $37^{\circ} \mathrm{C} .1 \mu \mathrm{M}$ Oregon green tubulin was added to the mixture every $30 \mathrm{~min}$ for $3-4 \mathrm{~h}$. Then $2 \mu \mathrm{l}$ of the Oregon green microtubules was added to $20 \mu \mathrm{l}$ of $0.3 \mu \mathrm{M}$ tubulin, $0.2 \mu \mathrm{M}$ rhodamine tubulin, $0.5 \mathrm{mM}$
GMPCPP in BRB80. The mixture was incubated at $37^{\circ} \mathrm{C}$ for $30 \mathrm{~min}$ and replenished with $0.3 \mu \mathrm{M}$ of unlabelled tubulin and $0.2 \mu \mathrm{M}$ of rhodamine tubulin every $30 \mathrm{~min}$ for $3-4 \mathrm{~h}$. As tubulin grows approximately 3 times faster from the plus- compared to the minus-end ${ }^{27}$, the red fragments at the minus-ends of the microtubules were visibly shorter than those at the plusends. The polarity of microtubules was confirmed in a kinesin gliding assay. Microtubule binding assays. $2 \mu \mathrm{l}$ of kinetochore beads was mixed with $2 \mu \mathrm{l}$ of capped microtubules. The mixture was incubated for $5 \mathrm{~min}$ at room temperature, diluted with $40 \mu \mathrm{l}$ of BRB80 and observed with a Zeiss Axioskop microscope and a 63× PlanApo 1.6 lens using a Colour Cool View camera (Photonic Sciences, East Sussex). To avoid bias, all microtubules containing a bead were photographed. To examine the microtubule colour to which the beads were bound, the colours were split into the three components of the redgreen-blue (RGB) signal. Alternatively, $1 \mu$ l of the GMPCPP microtubules was mixed with $10 \mu \mathrm{l}$ of the kinetochore beads, allowed to bind for $5 \mathrm{~min}$, then diluted with $200 \mu \mathrm{l}$ of BRB80 and observed the same way.

Data analysis. For the experiments investigating binding to GMPCPP versus GDP lattice, we counted only the beads that were visibly not bound to the end, so beads that obscured the microtubule end when bound were not counted. To normalize the bead numbers bound to either type of the lattice, the total numbers of beads bound to GMPCPP and GDP fragments were multiplied by the total lengths of GMPCPP and GDP fragments, so the percentage of beads bound to the GMPCPP fragments was $100 \times N_{\mathrm{c}} L_{\mathrm{d}} / N_{\mathrm{c}} L_{\mathrm{d}}+N_{\mathrm{d}} L_{\mathrm{c}}$, and the percentage of the beads bound to the GDP fragments was $100 \times N_{\mathrm{d}} L_{\mathrm{c}} / N_{\mathrm{c}} L_{\mathrm{d}}+N_{\mathrm{d}} L_{\mathrm{c}}$, where $N_{\mathrm{c}}$ is 67 (the number of beads bound to GMPCPP fragments), $N_{\mathrm{d}}$ is 8 (the number of beads bound to GDP fragments), $L_{\mathrm{c}}$ is $485 \mu \mathrm{m}$ (the total length of the GMPCPP fragments), and $L_{\mathrm{d}}$ is $539 \mu \mathrm{m}$ (the total length of the GDP fragments). Three independent experiments were done, and data added together and analysed. For the experiments on the effect of taxol addition, 62 beads were counted. To analyse the binding of beads to the plus-end, the minus-end and the lattice, we used GMPCPP microtubules. A microtubule was counted as binding to the end if it was obscuring the end of the microtubule. Only microtubules that had a clearly distinguishable polarity were included ( $\sim 90 \%)$. Three independent experiments were done, and all data are summarized in Fig. 3b, for a total of 292 beads. The distance from the microtubule minus-end to the centre of the bead bound was divided by the length of the microtubule and expressed as a percentage.

Received 11 April; accepted 30 May 1997

1. Rieder, C. L. \& Alexander, S. P. Kinetochores are transported poleward along a single astral microtubule during chromosome attachment to the spindle in newt lung cells. J. Cell Biol. 110, 81-95 (1990).

2. Skibbens, R. V., Skeen, V. P. \& Salmon, E. D. Directional instability of kinetochore motility during chromosome congression and segregation in mitotic newt lung cells: A push-pull mechanism. J. Cell. Biol. 122, 859-875 (1993).

3. Earnshaw, W. C. \& Tomkiel, J. E. Centromere and kinetomore structure. Curr. Biol. 4, 86-93 (1992).

4. Bloom, K. The centromere frontier: kinetochore components, microtubule-based motility, and the CEN-value paradox. Cell 73, 621-624 (1993).

5. Kirschner, M. W. \& Mitchison, T. J. Beyond self assembly: from microtubules to morphogenesis. Cell 45, 329-342 (1986).

6. Schilstra, M. J., Martin, S. R. \& Bayley, P. M. On the relationship between nucleotide hydrolysis and microtubule assembly: studies with a GTP-regenerating system. Biochem. Biophys. Res. Comm. 147, 588-595 (1987).

7. O’Brien, E. T., Voter, W. A. \& Erickson, H. P. GTP hydrolysis during microtubule assembly. Biochemistry 26, 4148-4156 (1987).

8. Stewart, R. J., Farrel, K. W. \& Wilson, L. Role of GTP hydrolysis in microtubule polymerisation: evidence for a coupled hydrolysis mechanism. Biochemistry 29, 6489-6498 (1990).

9. Hyman, A. A., Salser, S., Drechsel, D. N., Unwin, N. \& Mitchison, T. J. Role of GTP hydrolysis in microtubule dynamics: information from a slowly hydrolyzable analogue, GMPCPP. Mol. Biol. Cell. 3, $1155-1167$ (1992)

10. Sorger, P. K., Severin, F. F. \& Hyman, A. A. Factors required for the binding of reassembled yeast kinetochores to microtubules in vitro. J. Cell. Biol. 127, 995-1008 (1994).

11. Bailey, N. Statistical methods in Biology (Cambridge Univ. Press, 1995).

12. Hyman, A. A., Chretien, D., Arnal, I. \& Wade, R. H. Structural changes accompanying GTP hydrolysis in microtubules: information from a slowly hydrolyzable analogue guanylyl- $(\alpha, \beta)$-methylenediphosphonate. J. Cell Biol. 128, 117-125 (1995).

13. Arnal, I. \& Wade, R. H. How does taxol stabilize microtubules? Curr. Biol. 5, 900-908 (1995).

14. McIntosh, J. R. \& Euteneuer, U. Tubulin hooks as probes for microtubule polarity: an analysis of the method and an evaluation of data on microtubule polarity in the mitotic spindle. J. Cell Biol. 98, 525533 (1984).

15. Kingsbury, J. \& Koshland, D. Centromere-dependent binding of yeast minichromosomes to microtubules in vitro. Cell 66, 483-495 (1991).

16. Mitchison, T. J. \& Kirschner, T. J. Dynamic instability of microtubule growth. Nature 312, 237-242 (1984).

17. Lechner, J. \& Carbon, J. A $240 \mathrm{kd}$ multisubunit protein complex, CBF3, is a major component of the budding yeast centromere. Cell 64, 717-726 (1991).

18. Jiang, W., Lechner, J. \& Carbon, J. Isolation and characterization of a gene (CBF2) specifying a protein component of the budding yeast centromere. J. Cell Biol. 121, 513-519 (1993). 
19. Strunnikov, A. V., Kingsbury, J. \& Koshland, D. CEP3 encodes a centromere protein of Saccharomyces cerevisiae. J. Cell Biol. 128, 749-760 (1995).

20. Connely, C. \& Heiter, P. Budding yeast SKP1 encodes an evolutionary conserved protein required for cell cycle progression. Cell 86, 275-285 (1996).

21. Kingbury, J. \& Koshland, D. Centromere function on minichromosomes isolated from budding yeast. Mol. Biol. Cell 4, 859-870 (1993).

22. Miller, J. M., Wang, W., Balczon, R. \& Dentler, W. L. Ciliary microtubule capping structures contain a mammalian kinetochore antigen. J. Cell. Biol. 110, 703-714 (1990).

23. Wang, W., Suprenant, K. \& Dentler, W. Reversible association of a 97-kDa protein complex found at the tips of ciliary microtubules with in vitro assembled microtubules. J. Biol. Chem. 268, 24796-24807 (1993)

24. Mitchison, T., Evans, L., Schulze, E. \& Kirschner, M. Sites of microtubule assembly and disassembly in the mitotic spindle. Cell 45, 515-527 (1986).

25. Hyman, A. A. \& Mitchison, T. J. Two different microtubule-based motor activities with opposite polarities in kinetochores. Nature 351, 206-211 (1991).

26. Cassimeris, L., Pryer, N. K. \& Salmon, E. D. Real-time observations of microtubule dynamic instability in living cells. J. Cell Biol. 107, 2223-2231 (1988).

27. Walker, R. A. et al. Dynamic instability of individual microtubules analysed by video light microscopy: rate constants and transition frequencies. J. Cell Biol. 107, 1437-1448 (1988).

28. Belmont, L. D. Hyman, A. A., Sawin, K. E \& Mitchison, T. J. Real-time visualization of cell cycledependent changes in microtubule dynamics in cytoplasmic extracts. Cell 62, 579-589 (1990).

29. Sammak, P. J. \& Borisy, G. G. Direct observation of microtubule dynamics in living cells. Nature 332, 724-726 (1988)

30. Hyman, A. A. et al. Preparation of modified tubulins. Methods Enzymol. 196, 478-485 (1991).

31. Huitorel, P. \& Kirschner, M. W. The polarity and stability of microtubule capture by the kinetochore. J. Cell. Biol. 106, 151-160 (1988)

Acknowledgements. We thank S. Schuyler for early experiments suggesting the role of lattice structure J. Howard for help with statistics; and M. Glotzer, P. Gonczy, S. Eaton, K. Simons, R. Heald, S. Reinsch and S. Andersen for critical reading of the manuscript.

Correspondence and requests for materials should be addressed to A.A.H.

\section{Mutant analysis links the} translocon and BiP to retrograde protein

\section{transport for ER degradation} Richard K. Plemper ${ }^{\star}$, Sigrun Böhmler*, Javier Bordallo*,
Thomas Sommer ${ }^{\star}$ \& Dieter H. Wolf*

* Institut für Biochemie der Universität Stuttgart, Pfaffenwaldring 55, D-70569 Stuttgart, Germany

$\dagger$ Max-Delbrück-Centrum für Molekulare Medizin, Robert-Rössle Strasse 10,

D-13122 Berlin, Germany

Proteins enter the secretory pathway through the endoplasmic reticulum $^{1}$, which delivers properly folded proteins to their site of action $^{2}$ and contains a quality-control system to monitor and prevent abnormal proteins from being delivered ${ }^{3}$. Many of these proteins are degraded by the cytoplasmic proteasome $e^{4-8}$, which requires their retrograde transport to the cytoplasm ${ }^{5,6}$. Based on a co-immunoprecipitation of major histocompatibility complex (MHC) class I heavy-chain breakdown intermediates with the translocon subunit Sec61p (refs 9, 10), it was speculated that Sec61p may be involved in retrograde transport ${ }^{11}$. Here we present functional evidence from genetic studies that Sec61p mediates retrograde transport of a mutated lumenal yeast carboxypeptidase $y c s Y\left(\mathrm{CPY}^{\star}\right)$ in vivo. The endoplasmic reticulum lumenal chaperone BiP $(\operatorname{Kar} 2 p)$ and Sec63p, which are also subunits of the import machinery ${ }^{10,12}$, are involved in export of $\mathrm{CPY}^{\star}$ to the cytosol. Thus our results demonstrate that retrograde transport of proteins is mediated by a functional translocon. We consider the export of endoplasmic reticulum-localized proteins to the cytosol by the translocon for proteasome degradation to be a general process in eukaryotic cell biology.

Proteins cross the membrane of the endoplasmic reticulum (ER) either co-translationally or post-translationally ${ }^{9,10}$. For both translocation routes, the Sec61 heterotrimeric complex consisting of yeast proteins Sec61p, Sbh1p and Sss1p (ref. 10) constitutes the membrane channel. The post-translational pathway depends on an additional tetrameric complex consisting of the ER transmembrane proteins Sec62p, Sec63p and Sec71p, together with the peripheral membrane protein Sec 72p (refs 10, 12). Sec62p, Sec71p and Sec72p provide a cytosolic binding site for the newly synthesized precursor molecules, whereas Sec63p and the lumenal chaperone Kar2p (BiP) mediate the driving force for import ${ }^{10,12}$.

We analysed retrograde transport using yeast strains carrying mutant alleles of translocon components, and used mutated yeast

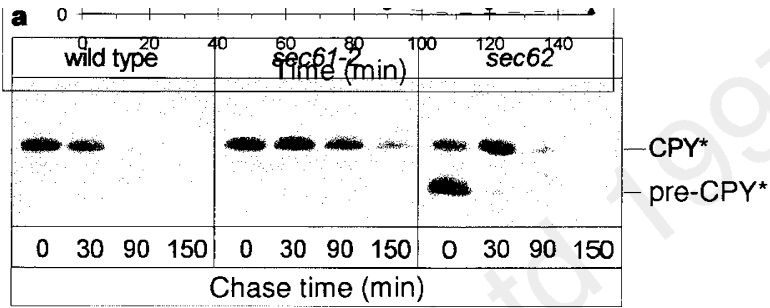

b
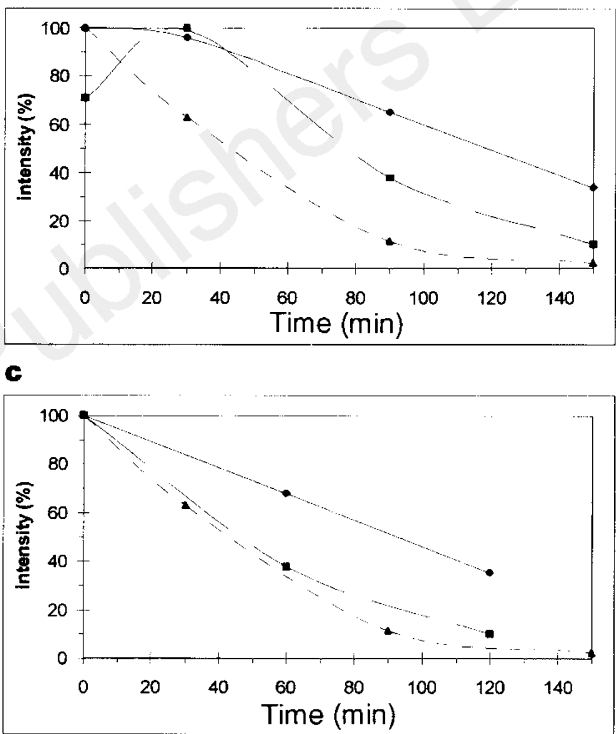

d
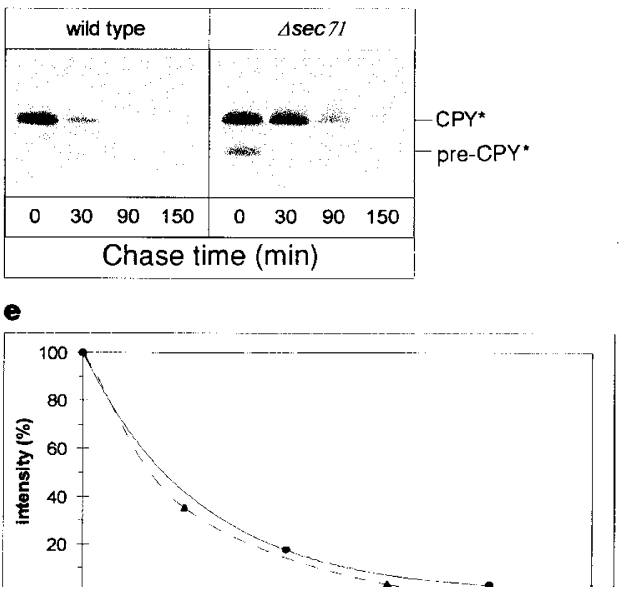

Figure $1 \mathrm{~A}$ functional Sec61p is required for ER degradation. a-c, CPY* is stabilized in the sec61-2 mutant, pre-CPY* import and not degradation is affected in the sec62 mutant. Pulse-chase analysis of $\mathrm{CPY}^{*}$ was performed using the isogenic strains W303-1C (wild type, triangles), YRP086 (sec61-2, diamonds) and YRP087 (sec62, squares). Cells were grown at the permissive temperature of $25^{\circ} \mathrm{C}$. After the indicated chase times the cells were lysed, CPY* was immunoprecipitated, and the antigenic material was separated by SDS-PAGE and analysed using a Molecular Dynamics imaging system. d, e, A sec71 knockout leads also to accumulation of pre-CPY* but not to a defect in $\mathrm{CPY}^{*}$ degradation. Pulse-chase analysis of the isogenic strains W303-1C (wild type, triangles) and YRP091 ( $\Delta$ sec 71 , circles) was done at $30^{\circ} \mathrm{C}$ as described in $\mathbf{a}$ 